
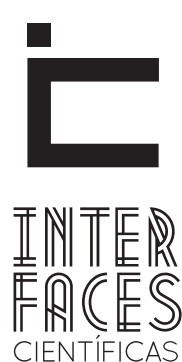

SA ÚDE E AMBIENTE

\title{
PROMOÇÃO E PREVENÇÃO DA SAÚDE DO HOMEM
}

Bibione Tercia de Oliveira Silva ${ }^{1}$ Mayanna Machado Freitas ${ }^{1}$

Gleice de Barros Santos Souza ${ }^{1}$
Mônica Nascimento Hardman ${ }^{1}$ Heliosania Clingea Fontes Sobral ${ }^{2}$

Ana Maria Laurindo da Silva ${ }^{3}$

\section{RESUMO}

O homem apresenta um conjunto de características próprias que são mantidas desde o período primitivo e a inclusão dos homens em ações de saúde é desafiadora, por estes não reconhecerem a importância do cuidado e a valorização do corpo no sentido da saúde como questões sociais. 0 enfermeiro, além de ser um profissional da saúde, atua como um educador onde desenvolve esclarecimentos de dúvidas e incentivando a população masculina com cuidados próprios. Nesse contexto, o objetivo do presente artigo foi investigar a ocorrência de políticas públicas voltadas para campanhas que conscientizem a população masculina e identificar medidas relacionadas à promoção e prevenção da saúde. Este trabalho consistiu em uma revisão bibliográfica, cuja estratégia de busca incluiu consulta às bases de dados eletrônicas Scielo, Science
Direct e Bireme. É necessário que os profissionais de saúde, em relação à saúde do homem, adotem formas diferentes de pensar, rompam crenças e valores para incorporar novos conceitos pertencentes a sua saúde, adequando as ações de prevenção, promoção, proteção e recuperação aos períodos contrários aos do trabalho deles. Além disso, quando houver procura nos serviços de saúde, devem ser aproveitados de diferentes formas para garantir que o homem crie o hábito rotineiro de cuidados e que diferentes meios sejam utilizados para alcançá-los através de ações de educação em prol da saúde.

\section{PALAVRAS-CHAVE}

Saúde do Homem. Enfermagem. Atenção Primária. 


\section{ABSTRACT}

The man has a set of characteristics that are maintained from the primitive period and the inclusion of men in health actions is challenging, for they do not recognize the importance of the care and recovery of the body in the direction of health as social issues. The nurse and a health professional acts as a teacher where he develops clarification of doubts and encouraging the male population with proper care. In this context, the objective of this article was to investigate the occurrence of public policies focused campaigns that aware the male population and identify measures related to health promotion and prevention. This work consisted of a bibliographical revision, whose search strategy included consulting the electronic database SciELO, Science Direct, Bireme and Bireme. It is necessary that health professionals, in relation to human health, to adopt different ways of thinking, break beliefs and values to incorporate new concepts pertaining to your health, adapting the actions of prevention, promotion, protection and recovery periods contrary to those of their work. In addition, when there is demand in health services should be used in different ways to ensure that the man makes a habit of routine care and that different means are used to achieve them through health education.

\section{KEYWORDS}

Men's Health. Nursing. Primary Care

\section{RESUMEN}

El hombre tiene un conjunto de características que se mantienen desde la época primitiva y la inclusión de éstos en acciones de salud es un reto, porque no reconocen la importancia del cuidado y la recuperación del cuerpo como problemas sociales. La enfermera es un profesional de la salud que actúa como un maestro donde que elucida las dudas y alerta a la población masculina para con el cuidado apropiado. En este contexto, el objetivo de este artículo fue investigar la ocurrencia de campañas y políticas públicas enfocadas a concienciar la población masculina, y identificar las medidas relacionadas con la promoción de la salud y prevención. Este trabajo consistió en una revisión bibliográfica, cuya estrategia de búsqueda incluyó consultar los datos electrónica SciELO, Science Direct, Bireme y Bireme. Es necesario que los profesionales de la sanidad, en lo que se refiere a la salud humana, adopten diferentes formas de pensar, rompan creencias y valores para incorporar nuevos conceptos relacionados con su salud, adaptando las acciones de prevención, promoción, protección y recuperación en períodos contrarios a los de su trabajo. Además, cuando hay demanda en servicios de salud, debiese utilizar de diferentes maneras para asegurarse de que el hombre se haga la rutina y que se utilicen de diferentes medios para alcanzarlos a través de la educación para la salud.

\section{PALABRAS CLAVE}

Salud de los Hombres. Enfermería. Atención Primaria 


\section{INTRODUÇ̃̃o}

O homem apresenta um conjunto de características próprias que são mantidas desde o período primitivo, onde a força, a invulnerabilidade, virilidade, trabalho e ser chefe de família se tornaram um dos fatores responsáveis pela desvalorização do seu autocuidado (SILVA et al., 2010; SILVA, 2010).

0 índice de homens na procura de atendimento no setor primário é inferior ao das mulheres, uma vez que estes só procuram serviços de saúde quando apresentam sintomatologia (ALBANO; BASÍLIO; NEVES, 2010). Essa busca se contrapõe com estudos realizados por Brasil (2009), que informa que a Atenção Primária é o principal contato dos pacientes com os serviços de saúde independentemente de raça, gênero ou idade.

Observa-se que os homens necessitam de políticas de atenção à saúde, as quais objetivam a promoção e prevenção em prol de uma atenção mais significativa das ações de saúde específicas ao homem e seu reconhecimento nas condições sociais, tornando-se consciente a vulnerabilidade de adquirir patologias propensas ao homem (NEVES, 2010).

Segundo Carrara et al. (2009), a proposta inclusiva dos homem em ações de saúde é desafiadora, por não terem reconhecimento do cuidado e valorização do corpo no propósito da saúde como questões sociais. 0 preconceito existente em relação ao gênero masculino é evidente, pois é pouco discutida e abordada, além de haver uma escassez de conhecimentos nas campanhas de saúde pública que não são voltadas a este segmento da população (ALBANO; BASÍLIO; NEVES, 2010).
A Unidade Básica de Saúde é o melhor local para educar e estimular a comunidade masculina a adotar hábitos preventivos. A prevenção é veiculada a uma ação orientada, cuja finalidade é não permitir que o indivíduo adoeça, e assim, possa ter melhor qualidade de vida. E para a prevenção e promoção da saúde da população masculina é necessário o envolvimento com informações importantes, buscando a incorporação de hábitos preventivos (SILVA et al., 2010).

A saúde masculina, por ser pouco debatida e abordada se comparada à saúde feminina, precisa ser objeto de investigações e reconhecimento social. 0 presente trabalho mostra a importância das necessidades de assistência e dos serviços destinados à saúde do homem para que os mesmos tenham uma atenção e que possam perpetuar uma prática saudável de vida.

Nesse contexto, percebe-se a necessidade de aumentar o nível de conscientização, quanto à prevenção, promoção e adesão do homem ao serviço primário de saúde, e o conhecimento da população masculina sobre os fatores de risco das doenças acometidas. E ainda, reduzir no sistema público de saúde as taxas de morbidade e mortalidade.

Desse modo, o artigo teve como objetivos: investigar a ocorrência de políticas públicas voltadas para campanhas que conscientizem a população masculina; identificar medidas relacionadas à promoção e prevenção da saúde, bem como seus riscos e susceptibilidade do homem às doenças e informar sobre as necessidades dos homens em ter hábitos saudáveis, fazer exames preventivos e até mesmo procurar um médico como procedimento de rotina. 


\section{METODOLOGIA}

Este trabalho consistiu em uma revisão bibliográfica, cuja estratégia de busca incluiu consulta à base de dados eletrônicas Scielo, Science Direct e Bireme. 0 alvo dessa busca foram estudos com abordagem da enfermagem na saúde do homem. Para seleção, buscou-se a combinação dos termos saúde do homem, enfermagem e Atenção Básica. A busca foi realizada no período compreendido entre fevereiro e maio de 2013.

\section{DESENVOLVIMENTO}

Os primeiros estudos sobre a saúde dos homens foram realizados por norte-americanos na década de 70. Estes foram incentivados pela teoria e política feminista, onde os homens eram considerados instrumentos, responsáveis pelos agravos à saúde dos outros, especialmente da mulher (SILVA, 2010).

A saúde do homem tem sido desenvolvida ao longo dos anos e ainda encontra-se em fase de implantação. Compreender a dinâmica desses programas pode ajudar ao profissional de Enfermagem a desenvolver ações que promovam atendimento com qualidade dessa demanda do homem jovem e adulto. Esse programa insere os aspectos de sua vida produtiva social e econômica, proporcionando hábitos saudáveis (FIGUEIREDO, 2005).

A ideia de que as Unidades de Atenção Primária a Saúde (UAPS) sejam serviços destinados para crianças, mulheres e idosos é bastante corriqueira. (ALBANO et al., 2010). Para Carrara et al. (2009), é desafiador incluir os homens em ações de saúde pela ausência do cuidado no sentido da saúde como ques-
Para a inclusão dos artigos no estudo, foram definidos critérios a fim de atender adequadamente aos objetivos estabelecidos.

Critérios para inclusão dos artigos: a) abordagem sobre saúde do homem; b) discussão sobre promoção e prevenção da saúde masculina.

Os textos foram analisados e sintetizados de forma crítica, a fim de discutir as informações obtidas que correspondiam especificamente ao tema pretendido para compor esta revisão.

tão social. Deve-se ter em vista que os homens vivem sete anos a menos que as mulheres e são mais propensos a doenças como câncer, colesterol, diabetes, doenças do coração e hipertensão arterial mais elevada (SILVA, 2010).

Esse fato é visto como a socialização dos homens, onde o cuidado não é visto como uma prática na esfera masculina. Fatores de resistência como horários de atendimento, medo de descoberta de doenças e a presença de mulheres tanto como demanda de atendimento quanto de profissionais assistentes, tornam os espaços da Unidade Básica de Saúde feminizados, o que cria uma barreira a mais na questão do cuidado (ALBANO et al., 2010; SILVA et al., 2010).

Para Furtado et al. (2012) os modelos de masculinidade afastam os homens do autocuidado, ausentando-se dos serviços de saúde. Os valores que envolvem a educação familiar colocam o homem como um ser provedor e protetor. Essa tradição gera modelos masculinos pouco envolvidos a práticas de autocuidado e pode estimular o comportamento. Esta cultura 
tende a gerar modelos masculinos pouco aderentes a práticas de autocuidado e pode estimular o comportamento hostil, e de descuido com ele mesmo.

Os índices de morbimortalidade causados na população masculina afetam uma faixa etária compreendida entre 25 e 29 anos. Geralmente, as condições severas e crônicas de saúde estão associadas a doenças do aparelho respiratório, tumores, hipertensão arterial e até mesmo causas externas, como acidentes e violência (ALBANO et al., 2010).

Segundo Leite et al. (2010), a procura diminuída da população masculina ao serviço de saúde dificulta 0 acesso à Atenção Básica, medidas preventivas, ações de promoção, proteção da saúde e prevenção das doenças. Foi realizado uma pesquisa por Silva et al. (2010), onde algumas enfermeiras tiveram que identificar os motivos de oposição do homem às ações de saúde. Elas perceberam que estão relacionados com a cultura masculina, a organização do serviço na Estratégia Saúde da Família (ESF), as condições socioeconômicas e seu desconhecimento das ações de Saúde Pública.

Há também o desconhecimento da importância das ações de prevenção e manutenção da saúde, uma vez que eles não se mostram sensíveis aos trabalhos educativos. Como exemplo tem-se o câncer de próstata que afeta a maior parte da população e estima-se que um em cada 12 homens seja diagnosticado ao logo da vida (JUNIOR; LIMA, 2009). No entanto, os homens ainda têm uma visão de que são fortes e invulneráveis, sendo este o pensamento que os impedem de praticar ações saudáveis (SILVA et al., 2010).

A ausência de um programa específico para a saúde do homem pode ser um dos principais motivos da não realização periódicas de exames, os quais podem prevenir e até minimizar doenças, através de um diagnóstico precoce (JUNIOR; LIMA, 2009). Nesse sentido, em 27 de agosto de 2009, o Ministério da Saúde criou a Política Nacional de Atenção Integral à Saúde do Homem, que tem por objetivo facilitar e maximizar o acesso dos homens a este serviço. Além disso, cria maneiras de aperfeiçoar a assistência oferecida, ocasionando uma mudança cultural (MS, 2009).

A crítica aumenta quando se compartilha a ideia de que a atenção primária não dispõe de profissionais capacitados para assistir a especificidade da saúde do homem, correlacionando situações de vida e de trabalho desta população com possíveis agravos à saúde e demarcando ações de proteção e de promoção à saúde. Fazem-se necessários um olhar direcionado aos homens, uma técnica e um conhecimento epidemiológico dos problemas que os acometem ou podem acometê-los, para assim, pensar em meios para o atendimento das necessidades de saúde (CARRARA et al., 2009).

O enfermeiro, além de ser um profissional da saúde, atua como um educador onde desenvolve esclarecimentos de dúvidas e age incentivando a população masculina a realizar cuidados próprios (ALBANO et al., 2010). Em estudo realizado por Leite et al. (2010) sobre a procura pelo atendimento, observou-se que os homens com ensino superior completo apresentaram maior capacidade de problematizar o tema, tendo ideias concretas, porém não colocando em prática esse conhecimento.

Foram questionados sobre o medo de adoecer desses pacientes e $57 \%$ revelaram esse sentimento, apresentado como justificativa à dependência de terceiros e ausência das atividades diárias que podem influenciar no sustento da família. Os demais relataram não temer a doença, pois acreditam ser um processo natural que todos estão suscetíveis em algum momento da sua vida (ALBANO et al., 2010).

Devido às adequações necessárias nos serviços de saúde, as equipes de enfermagem encontram dificuldades de implementar a política de saúde do homem em suas unidades de saúde da família. Falta capacitação dos profissionais para este atendimento, material 
didático sobre o assunto e as unidades de atenção a saúde necessitam de adequações (JULIÃO, WEIGELT, 2011).

É possível afirmar que por meio de formas preventivas, muitas doenças poderiam ser evitadas, mas a resistência masculina quanto à prevenção ainda é notória, tendo em vista alguns pensamentos conservadores e alguns hábitos de vida que não conseguem mudar. Isso é visível para ações que buscam mudanças comportamentais. E essa não procura de serviços de saúde pode ser reunida em dois grupos: um de barreiras socioculturais e outro de barreiras institucionais (RODRIGUES, RIBEIRO, 2012).

\section{CONSIDERAÇÕES FINAIS}

É necessário que os profissionais de saúde, em relação à saúde do homem, adotem formas diferentes de pensar, rompam crenças e valores para incorporar novos conceitos pertencentes a sua saúde, adequando as ações de prevenção, promoção, proteção e recuperação aos períodos contrários aos do trabalho deles.

Além disso, quando houver procura nos serviços de saúde, devem ser aproveitados de diferentes formas para garantir que o homem crie o hábito rotineiro de cuidados e que diferentes meios sejam utilizados para alcança-los através de ações de educação em prol da saúde.

\section{REFERÊNCIAS}

ALBANO, B.R.; BASİLIO, M.C.; NEVES, J.B. Desafios para a inclusão dos homens nos serviços de Atenção Primária à Saúde. Revista de Enfermagem Integrada, v.3, n.2, 2010;

BRASIL. Ministério da Saúde. Política Nacional de Atenção Integral à Saúde do Homem (princípios e diretrizes). Disponível em: <http://dtr2001.saude.gov.br/sas/PORTARIAS/Port2008/PT-09-CONS.pdf.> Acesso em: mar. 2013;

CARRARA, S; RUSSO A. Jane; FARO, L. A política de atenção à saúde do homem no Brasil: os paradoxos da medicalização do corpo masculino. Revista de Saúde Coletiva, v. 19, n. 3, 2009;

FIGUEIREDO, W. Assistência à saúde dos homens: um desafio para os serviços na atenção primária. Ciência e Saúde Coletiva, v.10, n.1, p.7-17, 2005;

FURTADO, M.S.; SILVA, P.A.S.; GUILHON, A.B.; SOUZA, N.V.; DAVID, H.M.S.L. A saúde do homem na visão dos enfermeiros de uma unidade básica de saúde. Revista Anna Nery, v.16, n.3, 2012;

JULIÃO, G.G.; WEIGELT, L.D. Atenção á Saúde do homem em unidades de Estratégia de Saúde da Família. Revista de Enfermagem UFSM, v.1, n.2, 2012; 
JUNIOR, Eduardo Alves Lima; LIMA, Hermínio de Sousa. Promoção da saúde masculina na atenção básica. Pesquisa em Foco, v.17, n.2, p.32-41, 2009;

LEITE, Denise Fernandes; FERREIRA, Iracema Maria G; SOUZA, Marta Solange de; NUNES, Vanessa Silva; CASTRO, Paulo Roberto de. A influência de um programa de educação na saúde do homem. 0 Mundo da Saúde, v.34, n.1, p. 50-56, 2010;

Ministério da Saúde (Brasil), Secretaria de Atenção à Saúde, Política Nacional de Humanização da Atenção e Gestão do SUS, 2009;

RODRIGUES, J.F.; RIBEIRO, E.R. O homem e a mudança de pensamento em relação à sua saúde. Caderno Saúde e Desenvolvimento, v.1, n.1, 2012;

SILVA, M.E.D.C.; ALVARENGA, W.A.; SILVA, S.S.; BARBOSA, L.D.C.S.; ROCHA, S.S. Resistência do homem às ações de saúde: percepção de enfermeiras da Estratégia Saúde da Família. Revista Interdisciplinar, v.3, n.3, 2010;

SILVA, S.O. Cuidado na perspectiva de homens: Um olhar da Enfermagem. Tese de mestrado em Enfermagem, Universidade Federal de Santa Maria, 2010. 